\title{
Fertility Preservation Counseling
}

National Cancer Institute

\section{Source}

National Cancer Institute. Fertility Preservation Counseling. NCI Thesaurus. Code C137829.

Professional guidance designed to provide information and address the emotional issues which arise in fertility preservation patients. 